\title{
Augmented Reality App to improve quality of life of people with cognitive and sensory disabilities
}

\author{
Mirko Rossi \\ Department of Computer, Control and \\ Management Engineering \\ Sapienza University of Rome \\ Rome, Italy \\ mirko.rossi@uniroma1.it
}

\author{
Mauro Grigioni \\ National Center for Technological \\ Innovation in Public Health \\ Istituto Superiore di Sanità \\ Rome, Italy \\ mauro.grigioni@iss.it
}

\author{
Giuseppe D'Avenio \\ National Center for Technological \\ Innovation in Public Health \\ Istituto Superiore di Sanità \\ Rome, Italy \\ giuseppe.davenio@iss.it
}

\author{
Sandra Morelli \\ National Center for Technological \\ Innovation in Public Health \\ Istituto Superiore di Sanità \\ Rome, Italy \\ sandra.morelli@iss.it
}

\begin{abstract}
In the last decades, number of people affected by cognitive decline is rising significantly. The main cause of this problem is believed to be the overall drift of the average population age. In general, cognitive impairment varies from mild to severe with mild represented as people may begin to notice a slight change in their cognitive functions while still be able to conduct their daily activities without major limitations; on the other hand, severe state of cognitive impairment leads to a progressive loss of comprehensive abilities as well as evaluating situations. These impairments may be represented by losing the ability to talk and write that leads to the inability to conduct an independent lifestyle. The decay of intellectual functions is associated with a progressive increase of sensory impairment (vision and hearing). In this paper we present a tool to help and support people who suffer from both mild agerelated cognitive decline and sensory impairment. We developed an augmented reality home-made App designed for smart glasses. This App would be a useful tool to provide more independence to patients during actual daily living activities and it will be able to notify users about a potentially dangerous situations, by providing cues in real time. In this way, elderly people, will be able continue their life at home for a longer time before having the need to move into an assisting living facility.
\end{abstract}

Keywords-multiple disability, augmented reality, smart glasses

\section{INTRODUCTION}

Sensory (visual and hearing) and cognitive impairments are highly prevalent among elderly people $(65+$ years old $)$ [1], this is due to the fact that, with aging, degenerative processes of organs physiology occur [2]. Moreover, the number of world population is growing and at the same time the balance is shifting to a higher percentage of older people [3]. Because of increasing in life expectancy [4], the prevalence is expected to increase over the upcoming decades [1].

Hearing impairment $(\mathrm{HI})$ are one of the most frequent sensory deficits among human population [5]. In 2012, World Health Organization (WHO) estimated that there are 360 million people around the world with hearing impairment (5.3\% of the world's population) of which about a third are over 65 years old [6]. However, the statistics is not well defined, in part due to the employment of different classification systems.

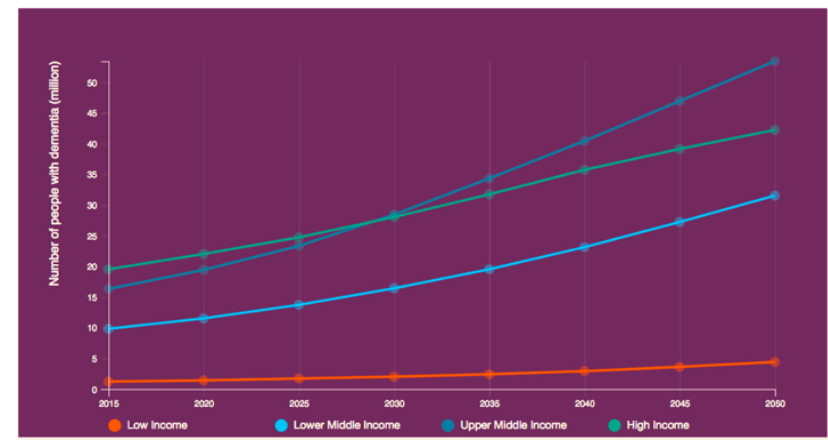

Fig. 1. Evolution of patients' number affected by dementia from 2015 to 2050. The countries are ranked in 4 classes based on their income (low, lower middle, upper middle and high).

Age-related hearing loss (ARHL) is primarily related to a functional loss of sensory and neural elements [2] with consequences in both cognitive and psychosocial spheres [7].

Visual impairment (VI) has a great importance for quality of life, socioeconomics, public health of societies and countries [8]. Approximately one over-65-person out of three are affected by visual-reducing eye disease [9]. In 2019, WHO has reported that at least 2.2 billion people worldwide have a visual impairment or blindness [10].

All around the world, about 46 million people suffer from dementia. This number is expected to increase to 131.5 million by 2050, mainly due to population aging [4] (Fig. 1). People with cognitive impairment are reported as being three times more hospitalized compared to individuals who are hospitalized for other conditions [11]. In addition, in many developed countries, healthcare costs have overtaken those related to cardiovascular diseases and cancer [12]. In general, cognitive decay starts with aging-related issues followed by a transition into a mild cognitive impairment status, leading ultimately to dementia. Mild cognitive impairment does not substantially affect daily function, whereas dementia causes cognitive changes that are potentially severe enough to affect daily function [13].

A worse quality of life is related to the above-mentioned targeted people [1]. In fact, people affected by these impairments depend on others and have difficulties conducting their daily life activities. 


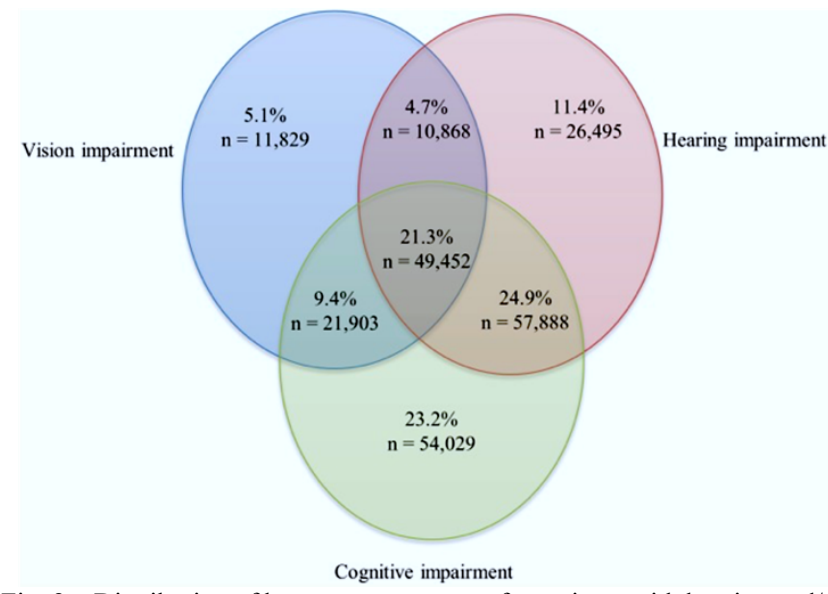

Fig. 2. Distribution of home care structures for patients with hearing and/or visual sensory and/or cognitive impairments. In this sample, $20.3 \%$ had none of these [1].

It was been demonstrated that an increased risk of cognitive decline and dementia is linked to age-related HIs [14] or VIs [15]. To date, however, research has mainly focused on single impairment (e.g., hearing, vision or cognitive impairment) and only few studies took into account their combined effects on the health of older people [1]. People affected by more than one of the previously mentioned disturbs (Fig. 2) are considered as vulnerable group who are at increased risk for unmet social, environmental, psychological and medical needs.

\section{A. ICT tools}

Elderly people should train their cognitive and motor skills on a daily basis. In fact, the risk of dementia increases with a lower participation in leisure activities carried out by the person [16]. Previous studies highlighted that a periodic physical and social activity helped maintaining neuronal plasticity and increasing brain activity among elderly people (e.g., [17]). One of the best ways to maintain neuronal functionality of elderly people for the longest period possible is to mitigate the degeneration by stimulating cognitive and physical functions [18]. The employment of Information and Communication Technologies (ICTs) in this area allows, in principle, to overcome these disadvantages [19]. As an application example of ICT tools, researchers evaluated the effectiveness of Serious Games as a training tool for cognitive abilities, obtaining satisfactory results in terms of cognitive and attention skills, reaction times, and a sense of well-being while playing computer games (e.g., [19]).

Some devices and systems are available to help and support individuals who are showing hearing issues to access the environment. Some devices convey information visually, and others enhance auditory information. Many assistive technological non-invasive devices are using light or vibration to alert users, while others are based on speech-totext technology. [20]

For people with VI several devices have been developed, which mostly use haptic or hearing technologies. For example, travelling aids, obstacle and object detectors which are scanning the environment, or assistive technologies for accessible information and communication, such as text-tospeech (TTS) software or braille conversion technology [20].

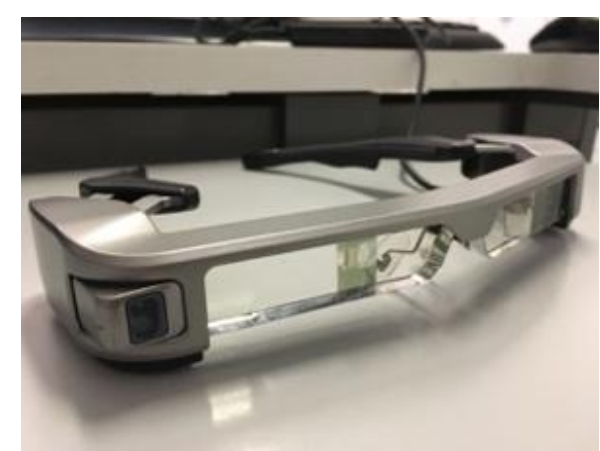

Fig. 3. The commercial HMD used in this study.

Recently, Virtual Reality (VR) and Augmented Reality (AR) technology are becoming an active frontier in digital communications. AR, in particular is a useful tool for supporting communication in people with disabilities. AR is a technology where a user's view or vision of the real world is enhanced or augmented with additional information generated from a computer model. The AR interface can decrease the cognitive load [21], [22], [23], [24], [25], [26]. In fact, elderly people are prone to use mental representations to enhance short-term memory [23]. In comparison to VR, $\mathrm{AR}$ is considered more user friendly, since it does not isolate the user from his/her environment. Furthermore, AR technology allows to combine cognitive and physical exercises.

For these reasons, we developed an $\mathrm{AR}$ marker-based Application to assist patients in their ADL - at home and also away from home.

\section{MATERIALS AND METHODS}

\section{A. Set-up}

To be run, the App requires only an HMD device, earphone and a printed image (marker) which we have associated with augmented information (Augmented Object, AO) via software. The HMD is equipped with a series of integrated sensors, including a camera embedded on its right side, and with TTS technology (Fig. 3). In order to program the markers to make them recognized by the HMD and associate each of them with an AO so that messages will be associated (in oral or textual form) to be provided to the user, we used Unity $3 \mathrm{~d}$, a cross-platform game engine, and a plugin provided by the HMD's manufacturer.

\section{B. User interface}

When the user wears the HMD, he experiences the surrounding real-world environment while the software is generating visual elements that are overlaid on the real-life visualization [27]. We have set specific markers on the physical objects of interest, as suggested by the National Institute on Aging (USA) [28]. Each of these markers is associated to a particular AO (text, picture, ...), which can be programmed based on each subject's preferences and needs. When a marker falls within the camera's field of view, the system shows the AO to the user, overlaid on the marker position. Furthermore, we decided to show on top of the HMD screen the fundamental user contacts (i.e.: police, ambulance and caregiver telephone number). Thus, if the user needs it, he/she can easily reach the emergency contact (e.g., police, ambulance or caregiver). Fig. 4 explains an example of programmed marker. The top panel shows the users' field of view when he/she puts on the HMD and the marker is out 


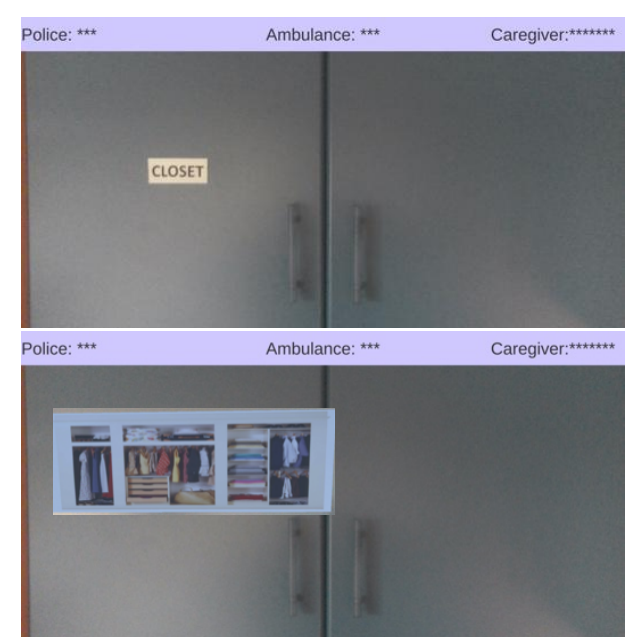

Fig. 4. Top image: the marker is applied on the closet door. Bottom image: when the user looks at the marker (the label), an image appears on the display representing the contents of the closet, without having to open it.

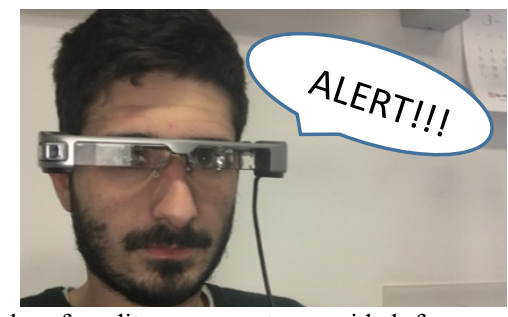

Fig. 5. Example of auditory prompts provided for people with visual impairment. The HMD will read out the message "Alert".

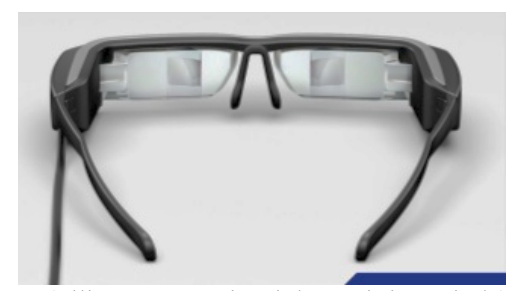

Fig. 6. Si-OLED (Silicon - Organic Light-Emitting Diode) display is able to show the visual prompts, useful for people affected by hearing impairment.

of the camera's field of view; on the bottom panel, the camera identifies the marker and the associated augmented objects are shown.

The HMDs can send and receive messages in the form of images, text, or audio. So, in the case of the HMD identifies or receives messages of potential dangerous situations, people with visual impairment will receive a message they are able to understand (an auditory cue through the earphone, Fig. 5), whereas people with auditory impairment will receive a visual cue (a text or an icon will appear on the display, Fig 6Finally, thanks to the camera being always active while the application is running, and to a mirroring App free-available on PlayStore ("WiFi-Display (miracast)"), which allows to share the user's field of view, the caregiver will be able to check at any time the patient's activity from his/her smartphone and therefore being able to quickly identify potentially dangerous situations.

\section{CONCLUSION AND FUTURE WORK}

The goal of the present paper was to present a tool able to support people with multiple impairments that are considered vulnerable with unique needs and challenges.
The AR-supported home environment can be useful for patients support while navigating the home itself. This patient empowerment should lead to an improved confidence during ADL, a higher degree of physical activity, and could enhance the social life.

Difficulties of older people with learning new skills as well as accessing new technological solutions would limit their ability to acquire the conceptual knowledge needed to interact with a novel interface, in both cognitive and sensory diseases. Furthermore, attentional control deficits would also disrupt their ability to adopt unfamiliar procedures.

The proposed system could improve the quality of life of targeted people. Major strength points of this tool are briefly: no need to learn new information or a high cognitive load neither any input device nor button is required to be pressed.

In conclusion, we are confident that our system could meet the criteria of compliance, dignity and usability, with the purpose to assess the latter, we plan to carry out an experimental study, initially involving elderly healthy people.

\section{ACKNOWLEDGMENT}

This study was supported by the project "High-end and Low-End Virtual Reality Systems for the Rehabilitation of Frailty in the Elderly" (Project Code: PE-2013-02355948), funded by Italian Ministry of Health.

\section{REFERENCES}

[1] D. M. Guthrie, J. G. S. Davidson, N. Williams, J. Campos, K. Hunter, P. Mick, J. B. Orange, M. K. Pichora-Fuller, N. A. Phillips, M. Y Savundranayagam, and W. Wittich, "Combined impairments in vision, hearing and cognition are associated with greater levels of functional and communication difficulties than cognitive impairment alone: Analysis of interRAI data for home care and long-term care recipients in Ontario," PLoS One, 13 (2), p. e0192971, 2018.

[2] T. N. Roth, D. Hanebuth, and R. Probst, "Prevalence of age-related hearing loss in Europe: A review," Euro Arch Otorhinolaryngol, 268:1101-1107, 2011.

[3] A. Suttie, A. Daly, G. Good, K. Mambetakunov, A. Orr, J. Scott, P. Techavachara, P. Verstraten, "Ageing and Visual impairment: a report by the Elderly Working Group of the World Blind Union," 2011.

[4] J. Hayhurst, "How Augmented Reality and Virtual Reality is Being Used to Support People Living with Dementia-Design Challenges and Future Directions" in Augmented Reality and Virtual Reality, Springer, pp. 295-305, 2018.

[5] C. D. Mathers, A. Smith, and M. Concha, "Global Burden of hearing loss in the year 2000," World Health Organization, Geneva, 2003.

[6] https://www.who.int/pbd/deafness/WHO_GE_HL.pdf

[7] D. Monzani, G. M. Galeazzi, E. Genovese, A. Marrara, and A. Martini, "Psychological profile and social behaviour of working adults with mild or moderate hearing loss," Acta Otorhinolaryngol Ital 28(2):6166, 2008.

[8] R. A. A. Bourne, J. B. Jonas, A. M. Bron, M. V. Cicinelli, A. Das, S. R. Flaxman, D. S. Friedman, J. E. Keeffe, J. H. Kempen, J. Leasher, H. Limburg, K. Naidoo, K. Pesudovs, T. Peto, J. Saadine, A. J. Silvester, N. Tahhan, H. R. Taylor, R. Varma, T. Y. Wong, and S. Resnikoff, "Prevalence and causes of vision loss in high-income countries and in Eastern and Central Europe in 2015: magnitude, temporal trends and projections," Br J Ophthalmol 102(5):575-585, 2018.

[9] D.A. Quillen, "Common Causes of Vision Loss in Elderly Patients", Am. Fam. Physician, 60(1):99-108, Jul 1999

[10] https:/www.who.int/news-room/fact-sheets/detail/blindness-andvisual-impairment

[11] Centers for Disease Control and Prevention, "Cognitive impairment: A call for action now!" U.S. Department of Health and Human Services, February 2011.

[12] M. Valenzuela and P. Sachdev, "Can Cognitive Exercise Prevent Onset of Dementia? Systematic Review of Randomized Clinical Trials with Longitudinal Follow-up," American Journal of Geriatric Psychiatry, 17(3), pp. 179-187, 2009.

[13] Canadian Task Force on Preventive Health Care, "Recommendations on screening for cognitive impairment in older adults," Canadian Medical Association Journal, 2016; 188:37-46.

[14] F. R. Lin, K. Yaffe, and J. Xia, Q. L. Xue, T. B. Harris, E. PurchaseHelzner, S. Satterfield, H. N. Ayonayon, L. Ferrucci, and E. M. 
Simonsick, "Hearing loss and cognitive decline in older adults," JAMA Intern Med, 173(4):293-299, 2013.

[15] S. P. Chen, J. Bhattacharya, and S. Pershing, "Association of vision loss with cognition in older adults," Journal of the American Medical Association Ophthalmology, 135(9):963-70, 2017.

[16] J. Verghese, R. B. Lipton, M. J. Katz, C. B. Hall, C. A. Derby, G. Kuslansky, A. F. Ambrose, M. Sliwinski, and H. Buschke, "Leisure activities and the risk of dementia in the elderly," N Engl J Med, 348:2508-2516, June 2013.

[17] A. Verdelho, S. Madureira, J. M. Ferro, H. Baezner, C. Blahak, A. Poggesi, M. Hennerici, L. Pantoni, F. Fazekas, P. Scheltens, G. Waldemar, A. Wallin, T. Erkinjuntti and D. Inzitari,"Physical activity prevents progression for cognitive impairment and vascular dementia: results from the LADIS (Leukoaraiosis and Disability) study," Stroke, 43(12): 3331-5, December 2012.

[18] Y. Lin, H. Mao, Y. Tsai, and J. Chou, "Developing a serious game for the elderly to do physical and cognitive hybrid activities", IEEE $6^{\text {th }}$ International Conference on Serious Games and Applications for Health (SeGAH), pp. 1-8, 2018.

[19] A. López-Martínez, S. Santiago-Ramajo, A. Caracuel-Romero, C. Valls-Serrano, M. J. Hornos, M. J. Rodríguez-Fórtiz, "Game of gifts purchase: Computer-based training of executive functions for the elderly", Proceeding of IEEE $1^{\text {st }}$ International Conference on Serious Games and Applications for Health (SeGAH 2011), pp. 1-8, 2011.

[20] http://www.europarl.europa.eu/RegData/etudes/IDAN/2018/603218/E PRS_IDA(2018)603218(ANN2)_EN.pdf

[21] E. Quintana and J. Favela, "Augmented Reality Annotations to Assist Persons with Alzheimer's and Their Caregivers", Personal and Ubiquitous Computing," vol. 17, no. 6, pp. 1105-1116, 2012.

[22] Y. J. Chang, H. Liu, Y. Kang, C. Chian, and Yao-Sheng Chang, "Using augmented reality smart glasses to design games for cognitive training," $13^{\text {th }}$ International Conference on Remote Engineering and Virtual Instrumentation (REV), 252, 2016.

[23] T. Chandrasekera, M. Kang, P. Hebert, and P. Choo, "Augmenting space: enhancing health, safety, and well-being of older adults through hybrid spaces," Technology and Disability 29(3):141-151, 2017.

[24] S. Wood and R. J. McCrindle, "Augmented reality, discovery and information system for people with memory loss," Personal and Ubiquitous Computing, 17(6), August 2013.

[25] C. Boletsis and S. McCallum, "Augmented Reality Cubes for Cognitive Gaming: Preliminary Usability and Game Experience Testing," Int. J. Serious Game, 3(1),3-18, 2016.

[26] L. Bonanni, C. H. Lee, and T. Selker, "Counter Intelligence: Augmented Reality Kitchen,” Proc. CHI, vol. 2239, p.44, 2005.

[27] B. Aruanno, F. Garzotto, and M. C. Rodriguez, "HoloLens-based Mixed Reality Experiences for Subjects with Alzheimer's Disease," In Proceedings of the $12^{\text {th }}$ Biannual Conference on Italian SIGCHI Chapter (CHItaly '17), Article 15, 9 pages, September 2017.

[28] https://www.nia.nih.gov/health/home-safety-and-alzheimers-disease 